\title{
Video Article \\ Scalable Quantum Integrated Circuits on Superconducting Two-Dimensional Electron Gas Platform
}

\author{
Kaveh Delfanazari ${ }^{1,2}$, Pengcheng $\mathrm{Ma}^{2}$, Reuben Puddy², Teng Yi ${ }^{2}$, Moda Cao ${ }^{2}$, Yilmaz Gul ${ }^{3}$, Carly L. Richardson ${ }^{4}$, lan Farrer ${ }^{2,5}$, David Ritchie ${ }^{2}$, \\ Hannah J. Joyce ${ }^{1}$, Michael J. Kelly ${ }^{1,2}$, Charles G. Smith ${ }^{2}$ \\ ${ }^{1}$ Centre for Advanced Photonics and Electronics, Engineering Department, University of Cambridge \\ ${ }^{2}$ Department of Physics, Cavendish Laboratory, University of Cambridge \\ ${ }^{3}$ Department of Electronic and Electrical Engineering, University College London \\ ${ }^{4}$ Department of Materials Science and Metallurgy, University of Cambridge \\ ${ }^{5}$ Department of Electronic and Electrical Engineering, University of Sheffield
}

Correspondence to: Kaveh Delfanazari at kd398@cam.ac.uk

URL: https://www.jove.com/video/57818

DOI: doi: $10.3791 / 57818$

Keywords: Engineering, Issue 150, two-dimensional Josephson junctions, quantum processing, quantum integrated circuits, hybrid superconductorsemiconductor devices, topological superconductivity, Majorana devices

Date Published: 8/2/2019

Citation: Delfanazari, K., Ma, P., Puddy, R., Yi, T., Cao, M., Gul, Y., Richardson, C.L., Farrer, I., Ritchie, D., Joyce, H.J., Kelly, M.J., Smith, C.G. Scalable Quantum Integrated Circuits on Superconducting Two-Dimensional Electron Gas Platform. J. Vis. Exp. (150), e57818, doi:10.3791/57818 (2019).

\section{Abstract}

To form a coherent quantum transport in hybrid superconductor-semiconductor (S-Sm) junctions, the formation of a homogeneous and barrierfree interface between two different materials is necessary. The S-Sm junction with high interface transparency will then facilitate the observation of the induced hard superconducting gap, which is the key requirement to access the topological phases (TPs) and observation of exotic quasiparticles such as Majorana zero modes (MZM) in hybrid systems. A material platform that can support observation of TPs and allows the realization of complex and branched geometries is therefore highly demanding in quantum processing and computing science and technology. Here, we introduce a two-dimensional material system and study the proximity induced superconductivity in semiconducting two-dimensional electron gas (2DEG) that is the basis of a hybrid quantum integrated circuit (QIC). The 2DEG is a $30 \mathrm{~nm}$ thick $\ln _{0.75} \mathrm{Ga}_{0.25} \mathrm{As}$ quantum well that is buried between two $\ln _{0.75} \mathrm{Al}_{0.25} \mathrm{As}$ barriers in a heterostructure. Niobium (Nb) films are used as the superconducting electrodes to form $\mathrm{Nb}$ $\mathrm{In}_{0.75} \mathrm{Ga}_{0.25} \mathrm{As}-\mathrm{Nb}$ Josephson junctions (JJs) that are symmetric, planar and ballistic. Two different approaches were used to form the JJs and QICs. The long junctions were fabricated photolithographically, but e-beam lithography was used for short junctions' fabrication. The coherent quantum transport measurements as a function of temperature in the presence/absence of magnetic field $B$ are discussed. In both device fabrication approaches, the proximity induced superconducting properties were observed in the $\ln _{0.75} G_{0} a_{0.25} A s 2 D E G$. It was found that e-beam lithographically patterned JJs of shorter lengths result in observation of induced superconducting gap at much higher temperature ranges. The results that are reproducible and clean suggesting that the hybrid $2 \mathrm{D}$ JJs and QICs based on $\operatorname{In}_{0.75} \mathrm{Ga}_{0.25}$ As quantum wells could be a promising material platform to realize the real complex and scalable electronic and photonic quantum circuitry and devices.

\section{Video Link}

The video component of this article can be found at https://www.jove.com/video/57818/

\section{Introduction}

A Josephson junction $(\mathrm{JJ})$ is formed by sandwiching a thin layer of a non-superconducting (normal) material between two superconductors ${ }^{1}$. Various novel quantum electronic and photonic circuits and devices can be built based on JJs $\mathrm{J}^{2,3,4,5,6,7,8,9,10,11,12,13,14,15,16}$. Among them, the JJs with semiconductor as their non-superconducting (normal) part, or superconductor-semiconductor-superconductor (S-Sm-S) JJs, have received much attention in recent years after the purported detection of exotic Majorana particles with zero electrical charges at the interface

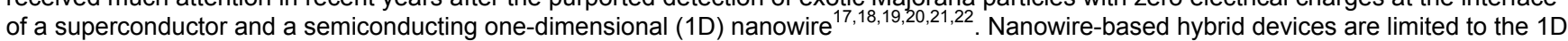
geometry of the nanowire and fabrication of $Y$ and/or T-structures out of them - a necessary requirement for Majorana braiding- is challenging ${ }^{22}$. The fine tuning of nanowire's chemical potential, for accessing topological phases, requires JJs with several electrostatically gates which causes quite a lot of issues in complex device fabrication out of nanowires. To overcome the scalability issues of 1D wires, two-dimensional (2D) material platforms are highly desirable ${ }^{19,22}$.

Among 2D materials, the two-dimensional electron gas (2DEG) platform -forms when electrons are confined to an interface between two different materials in a semiconductor heterostructure- is the most promising candidate ${ }^{22}$. The combination of 2DEG with superconductors and forming hybrid 2D JJs opens a new avenue towards the development of next-generation scalable quantum systems such as topological quantum processing and computing. They can support phase coherent quantum transport, and proximity induced superconductivity with high transmission probability, which are fundamental requirement for topological phase observation. In this regard, we demonstrate a QIC on a chip which consists of array of ballistic $2 \mathrm{D}$ JJs that can be controlled by 20 wires. Each junction has two Nb electrodes as the superconducting part 
and $\mathrm{In}_{0.75} \mathrm{Ga}_{0.25} \mathrm{As}$ quantum wells in a semiconducting heterojunction as the normal part. The wafer can be easily patterned to form complex structures and networked QICs.

The advantages of $\ln _{0.75} \mathrm{Ga}_{0.25}$ As $2 \mathrm{DEG}$ include: (i) relatively large $g$-factor, (ii) strong Rashba spin-orbit coupling, (iii) the low electron effective mass, and (iv) that the indium composition can be tuned allowing the formation of JJs with high interface transparency ${ }^{23,24,25}$. The wafer can be grown as a disk of up to $10 \mathrm{~cm}$ dimeter, allowing fabrication of thousands of hybrid $2 \mathrm{D} \mathrm{JJs}$ and complex QICs networks so overcoming the scalability challenges of these quantum devices.

We discuss two different approaches for device fabrications: For device 1, a circuit which includes eight identical and symmetric JJs of 850 $\mathrm{nm}$ length and $4 \mu \mathrm{m}$ widths are patterned by photolithography ${ }^{23,24}$. The device 2 includes eight junctions with different lengths. They all have the same width of $3 \mu \mathrm{m}$. The JJs are patterned by e-bam lithography ${ }^{25}$. The transport measurements at sub-Kelvin temperature ranges in absence/presence of magnetic field will be presented. The on-chip QICs consists of array of $2 \mathrm{D} \mathrm{Nb}-\operatorname{In}_{0.75} \mathrm{Ga}_{0.25} \mathrm{As}-\mathrm{Nb} \mathrm{JJ}$ s. The long and short junctions are measured in a dilution fridge with a base temperature of $40 \mathrm{mK}$ and liquid ${ }^{3} \mathrm{He}$ cooled cryostat with a base temperature of 300 $\mathrm{mK}$, respectively. Devices are biased with an ac-signal of $5 \mu \mathrm{V}$ at $70 \mathrm{~Hz}$ which is superimposed to the junction $d c$ voltage bias. A two-terminal standard lock-in technique is used to measure the device output ac-current ${ }^{23,24,25}$.

\section{Protocol}

NOTE: Semiconductor heterostructure and hybrid S-Sm Josephson junction fabrication are presented.

\section{Semiconducting heterostructure fabrication}

NOTE: The molecular beam epitaxy (MBE) grown $\ln _{0.75} \mathrm{Ga}_{0.25}$ As quantum wells are used in this study ${ }^{23,24,25,26}$. Figure 1 depicts the sequence of distinct layers:

1. Clean a $500 \mu \mathrm{m}$ thick, 3-inch semi-insulating (001) GaAs substrate and remove the oxide layer in high temperature (above $\left.200{ }^{\circ} \mathrm{C}\right)^{26}$.

2. Adjust the growth temperature at $580^{\circ} \mathrm{C}$ and grow the buffer layer of GaAs/AlAs/GaAs films with thicknesses of $50 / 75 / 250 \mathrm{~nm}{ }^{26}$.

3. Ramp down the substrate temperature for $20 \mathrm{~min}$ and then grow a step-graded buffer layer of InAIAs with a $1300 \mathrm{~nm}$ thickness at starting substrate temperatures of $T=416,390,360,341,331$ and $337^{\circ} \mathrm{C}^{26}$.

4. Grow a $30 \mathrm{~nm}$ thick $\ln _{0.75} \mathrm{Ga}_{0.25}$ As quantum well $2 \mathrm{DEG}$ at slightly higher substrate temperature ${ }^{26}$.

5. Cover the $2 \mathrm{DEG}$ quantum well with a $60 \mathrm{~nm} \mathrm{In}_{0.75} \mathrm{Al}_{0.25} \mathrm{As}$ spacer, and then modulation dope the wafer by a $15 \mathrm{~nm}$ thick of a n-type $\mathrm{In}_{0.75} \mathrm{Al}_{0.25} \mathrm{As}$. This will assure the conductance in the dark ${ }^{26}$.

6. Grow a $45 \mathrm{~nm} \ln _{0.75} \mathrm{Al}_{0.25}$ As layer followed by a cap layer of InGaAs with thickness of $2 \mathrm{~nm}^{26}$

7. Perform the measurement of the Shubnikov-de Haas oscillations and Hall effect to find the electron density $\left(n_{\mathrm{s}}\right)$ and mobility $\left(\mu_{\mathrm{e}}\right)$ at temperature $T=1.5 \mathrm{~K}^{26}$. From the transport measurements, it was inferred that the $n_{\mathrm{s}}=2.24 \times 10^{11}\left(\mathrm{~cm}^{-2}\right)$ and $\mu_{\mathrm{e}}=2.5 \times 10^{5}(\mathrm{~cm} / \mathrm{Vs})$ in the dark but $n_{\mathrm{s}}=2.28 \times 10^{11}\left(\mathrm{~cm}^{-2}\right)$ and $\mu_{\mathrm{e}}=2.58 \times 10^{5}\left(\mathrm{~cm}^{2} / \mathrm{Vs}\right)$ after illumination.

\section{Two-dimensional Josephson junction fabrication}

NOTE: Here, the fabrication process of the hybrid QICs with two different approaches are discussed ${ }^{23,24,25}$. Device 1 with eight identical long Josephson junctions was fabricated only with a few steps of photolithography processing. The second device fabrication procedure was similar to device 1 up to formation of JJs which step the e-beam- lithography was used.

1. Sketch the JJs and QIC device layout, including mesa and ohmic patterns by using AutoCad software ${ }^{25}$. Start the drawing by selecting appropriate layers to form the layer selector menu. Create a new layer from Format | Layer in the AutoCad software.

2. Design and fabricate the photolithography mask. Choose desired shapes and geometries from the panel menu in the software. Click on the desired shape of JJs (i.e., rectangles, squares) and push the drawing window to initiate the shape (click in the Autocad software help menu for more details).

3. Pattern the JJs and QICs designs, after developing the photoresist on the wafer, and fabricate the mesa structures to act as the active region (the raised area in Figure 1) by wet-etch in acid solutions of $\mathrm{H}_{2} \mathrm{SO}_{4}: \mathrm{H}_{2} \mathrm{O}_{2}:(1: 8: 1000)^{23,24,25}$. Rinse the device in DI water for $30 \mathrm{~s}$ and then dry with nitrogen gas.

4. Ensure an etch depth of $\sim 150 \mathrm{~nm}$ by the DEKTAK surface profiler ${ }^{23,24,25}$.

5. Form ohmic contacts, to make electrical contact between the metal and 2DEG, by spinning photoresist on top of the wafer and then exposure to UV light through a photo-mask. Develop the resist in MF-319 for $1 \mathrm{~min}$. Deposit a thin layer, between $50 \mathrm{~nm}$ and $100 \mathrm{~nm}$ of gold/ germanium/nickel (AuGeNi) alloy over the resist-patterned sample ${ }^{23,24,25}$

6. Etch a \# $140 \mathrm{~nm}$ deep trench on top of the active region to form 2D JJs by either photolithographically (device 1) or e- beam lithographically (device 2) patterning and wet-etching in acid described above (the JJs should be formed far from the ohmic contacts, a distance of $>100 \mu \mathrm{m}$, to ensure that the normal electrons from this part do not influence to the junction's interfaces) ${ }^{23,24,25}$.

7. Sputter a $\# 130 \mathrm{~nm}$ superconducting $\mathrm{Nb}$ film to form $\mathrm{Nb}-\mathrm{In}_{0.75} \mathrm{Ga}_{0.25} \mathrm{As}-\mathrm{Nb} \mathrm{JJs}$ (by DC magnetron sputtering in Ar plasma),

8. Deposit $10 / 50 \mathrm{~nm}$ thick Ti/Au films for electrical contacts and transport measurement purposes.

9. Transfer and load the device on the standard leadless chip carrier (LCC) by using GE varnish, and make the electrical contacts between the device and LCC pads by using gold wires.

10. Load the devices into ${ }^{3} \mathrm{He}$ cryostat or dilution refrigerator for transport measurements.

\section{Representative Results}

Figure 2a shows the scanning electron microscope (SEM) image of the device 1. A quantum circuit with 20 electrical wires can be seen. The design allows the measurement of one or series of JJs on a chip in one fridge cool-down. The SEM image of one junction on the circuit of Device 
2, that was fabricated by e-beam lithography, is shown in Figure $\mathbf{2 b}$. The distance between two $\mathrm{Nb}$ films in each side of the $\mathrm{Nb}-\mathrm{In}_{0.75} \mathrm{Ga}_{0.25} \mathrm{As}-\mathrm{Nb}$ junction is $L=550 \mathrm{~nm}$ at the shortest path. Figure 2c shows the SEM image of one junction of Device 1- which is photolithographically fabricated. Here, the two $\mathrm{Nb}$ electrodes are separated by a distance of $L=850 \mathrm{~nm}$.

The Blonder-Tinkham-Klapwijk (BTK) theory is an acceptable model to describe the quantum transport in hybrid S-Sm junctions ${ }^{27}$. The influence of the superconductor order parameters in semiconducting 2DEG results in a nonlinear differential conductance. At low temperatures, there are two possible reflection mechanisms at the $\mathrm{Nb}-\mathrm{In}_{0.75} \mathrm{Ga}_{0.25} \mathrm{As}$ interfaces: normal reflection which causes no charge transmission through the interface and the Andreev reflections, which transmits two charge quanta $2 e$, with the retroreflection of a hole ${ }^{23,24,25}$. As the superconducting condensate consists of spin singlet Cooper pairs, the reflected hole has the opposite spin as the incoming electron. The cartoon diagram of these two processes is shown in Figure $3 \mathbf{a}, \mathbf{b}$, respectively ${ }^{28}$.

If the interface between the $\mathrm{Nb}$ and $\ln _{0.75} \mathrm{Ga}_{0.25} \mathrm{As}$ contact is not transparent, there is coexistence of both normal and Andreev reflected electrons. Thus, the resistance increases and a zero-bias peak within the gap is formed. Such an in-gap peak in the $d V / d l\left(V_{\mathrm{SD}}\right)$ is not observed in our junctions. However, for a homogeneous and barrier free $(Z=0)$ interface between the $\mathrm{Nb}$ film and $\operatorname{In}_{0.75} \mathrm{Ga}_{0.25} \mathrm{As}$ contact, all incident electrons undergo Andreev reflection. In such condition, an excess current $l_{\text {exc }}$ is formed in the junction due to correlations of electron- and holelike quasiparticles. Therefore, the differential resistance within the gap is reduced and a flat U-shape dip in $d V / d l\left(V_{S D}\right)$ is observed. According to BTK model, it can be inferred that no tunneling barrier formed at the $\mathrm{Nb}-\mathrm{In}_{0.75} \mathrm{Ga}_{0.25} \mathrm{As}$ interfaces of both devices. Therefore, the barrier strength is estimated to be $Z<0.2$ in our junctions ${ }^{23,24,25}$.

Because of the proximity effect, induced gap of approximately $\Delta_{\text {ind }} \approx 100 \mu \mathrm{eV}$, and $650 \mu \mathrm{eV}$ are measured in the devices 1 and 2 , respectively. The temperature dependence induced superconducting gap with pronounced subharmonic energy gap structures (SGS) peaks and dips for device 1 are shown in Figure 4a. The multiple Andreev reflections (MAR) at the interfaces of the $N b-\ln _{0.75} \mathrm{Ga}_{0.25} \mathrm{As}$ junction result in the observation of SGS in the differential conductance. At the lowest measured temperature $T=50 \mathrm{mK}$ (red curve), the SGS appears with three peaks (named as P1, P2 and P3) and three dips (named as d1, d2 and d3). The temperature evolution of the peaks and dips due to the suppression of the induced superconductivity with temperature increase are shown in Figure $\mathbf{4 b}$. The SGS peak positions obey the expression $V=2 \Delta$ /ne ( $\Delta$ is the Nb gap energy, $n=1,2,3, \ldots$ is an integer, and $e$ is the electron charge): P1, P2, P3 and P4 positions approximately correspond to $2 \Delta / 3 \mathrm{e}, 2 \Delta / 4 \mathrm{e}, 2 \Delta / 6 \mathrm{e}$ and the induced gap edge but the dip positions do not follow the expression. All features are significantly temperature dependent, and the strongest (weakest) SGS peaks (dips) are observed at $T=50 \mathrm{mK}(800 \mathrm{mK})$. It is worth mentioning that even at temperatures above $T=500 \mathrm{mK}$ where the supercurrent can no longer be seen, the SGS is observed but it disappears at $T>800 \mathrm{mK}$ - when induced superconductivity is washed out.

For this device with array of eight $2 \mathrm{D} \mathrm{JJs}$, in 4 out of 7 junctions, a hard-induced superconducting gap in $\ln _{0.75} \mathrm{Ga}_{0.25} \mathrm{As} 2 \mathrm{DEG}$ was found ${ }^{23,24}$. However, three junctions showed a soft gap signature and neither a hard- nor a soft-gap structure was observed for the last junction because of a wire contact failure between the device and pad.

The superconducting gap as a function of applied $V_{S D}$ voltage and temperature of device 2 is shown in Figure 5a. This device was measured at a ${ }^{3} \mathrm{He}$ cryostat with base temperature of $T=280 \mathrm{mK}$. The temperature and magnetic field dependences transport measurements of device 2 do not show any sign of in-gap or sub-gap oscillations which are observed for device 1 (see Figure $\mathbf{5 a}, \mathbf{b}$ ). This could be due to the arrow-shaped geometry of the junction which may cause destructive interference of the MAR. Such features might appear in the differential conductance if the device is measured at much lower temperatures (dilution fridge base temperature). The induced gap is suppressed and moved toward zero voltage bias and their amplitudes diminish with further increasing of the applied temperature and magnetic field.

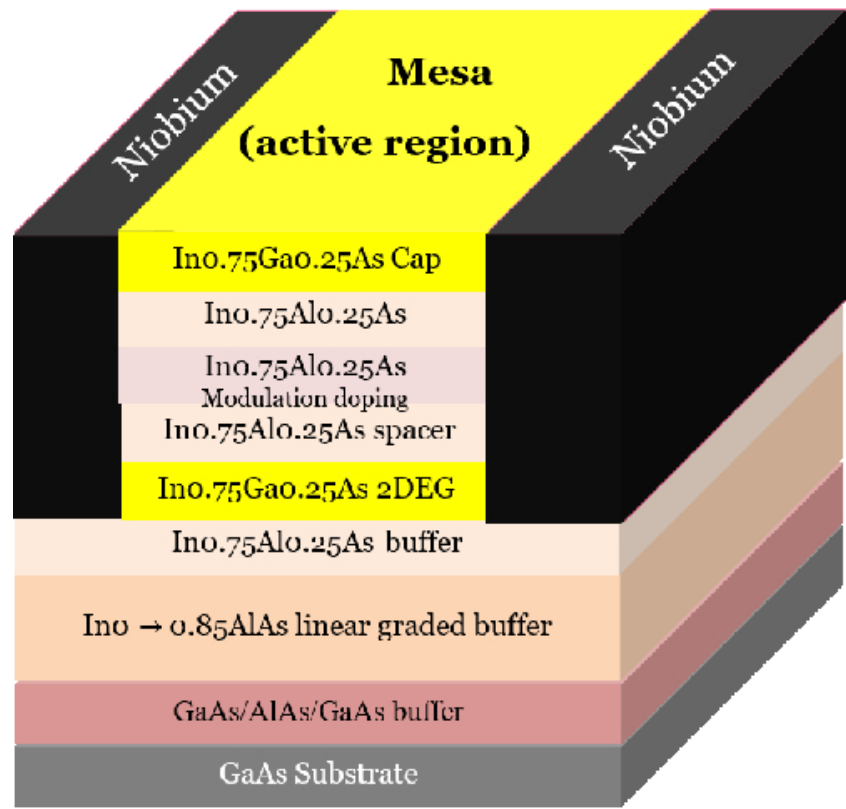

Figure 1. $\ln _{0.75} G_{0.25} A s / \ln _{0.75} \mathrm{Al}_{0.25} \mathrm{As} / \mathrm{GaAs}$ heterostructure. The schematic view of the heterojunction where an $\mathrm{In}_{0.75} \mathrm{Ga}_{0.25} \mathrm{As}$ quantum well with $30 \mathrm{~nm}$ thickness is formed $\# 120 \mathrm{~nm}$ below the wafer surface. Nb was used as the superconducting contacts (shown in black) to form a hybrid and ballistic $\mathrm{Nb}-\mathrm{In}_{0.75} \mathrm{Ga}_{0.25} \mathrm{As} 2 \mathrm{DEG}-\mathrm{Nb}$ Josephson junction. Please click here to view a larger version of this figure. 

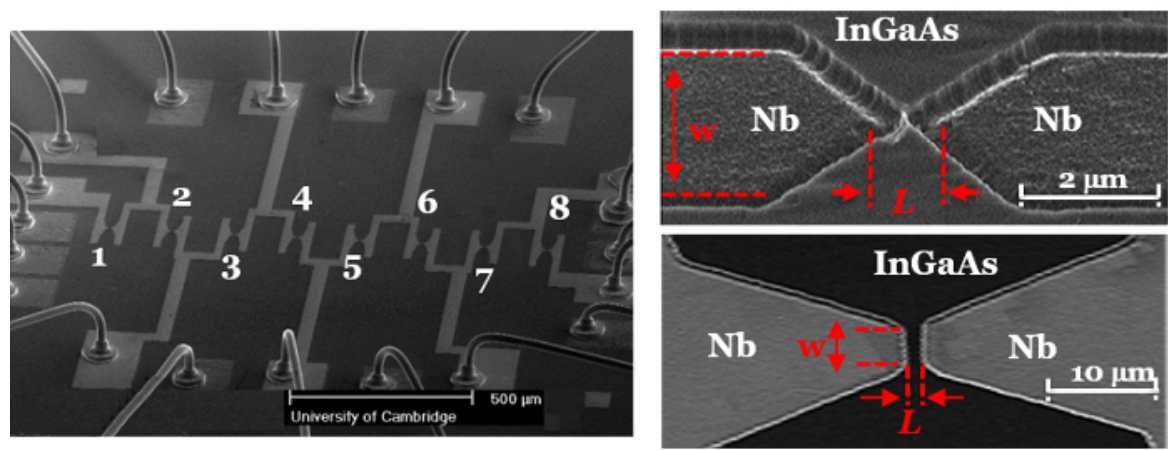

Figure 2: On-chip hybrid superconducting-semiconducting quantum circuits. (a) SEM image of the QICs device showing a top view of a quantum circuit with 20 control wires, and 8 planar and symmetric JJs on a chip. The SEM image of Nb-In $\mathrm{n}_{0.75} \mathrm{Ga}_{0.25} \mathrm{As}-\mathrm{Nb} \mathrm{JJ}$ sith an $\mathrm{In}_{0.75} \mathrm{Ga}_{0.25}$ As $2 \mathrm{DEG}$ gap of length $L=550 \mathrm{~nm}$ and $850 \mathrm{~nm}$ for e-beam lithographically (b) and photolithographically (c) fabricated junctions. Please click here to view a larger version of this figure.

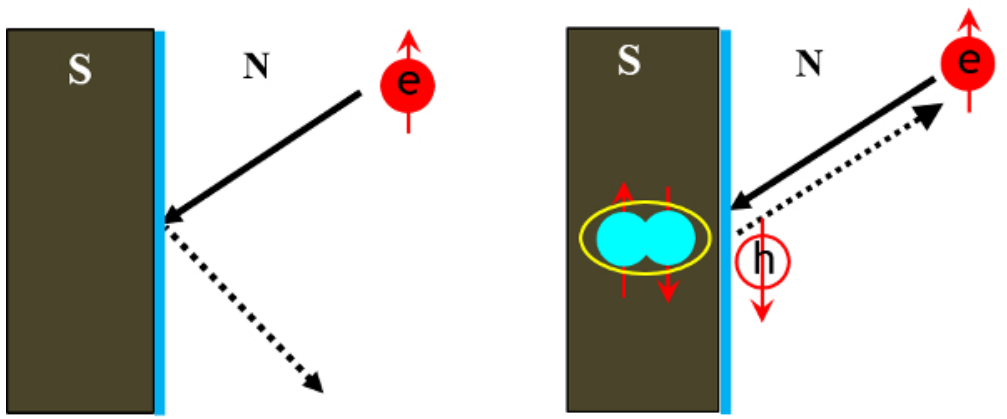

Figure 3. Normal and Andreev Reflections in hybrid superconducting-semiconducting junctions. (a) Specular quasiparticle reflection with no charge transmission through the interface. (b) Andreev reflection whereas the incoming electron is reflected as a hole in the opposite spin sub-band and transfer $2 \mathrm{e}$ charge into superconducting electrode. Please click here to view a larger version of this figure.
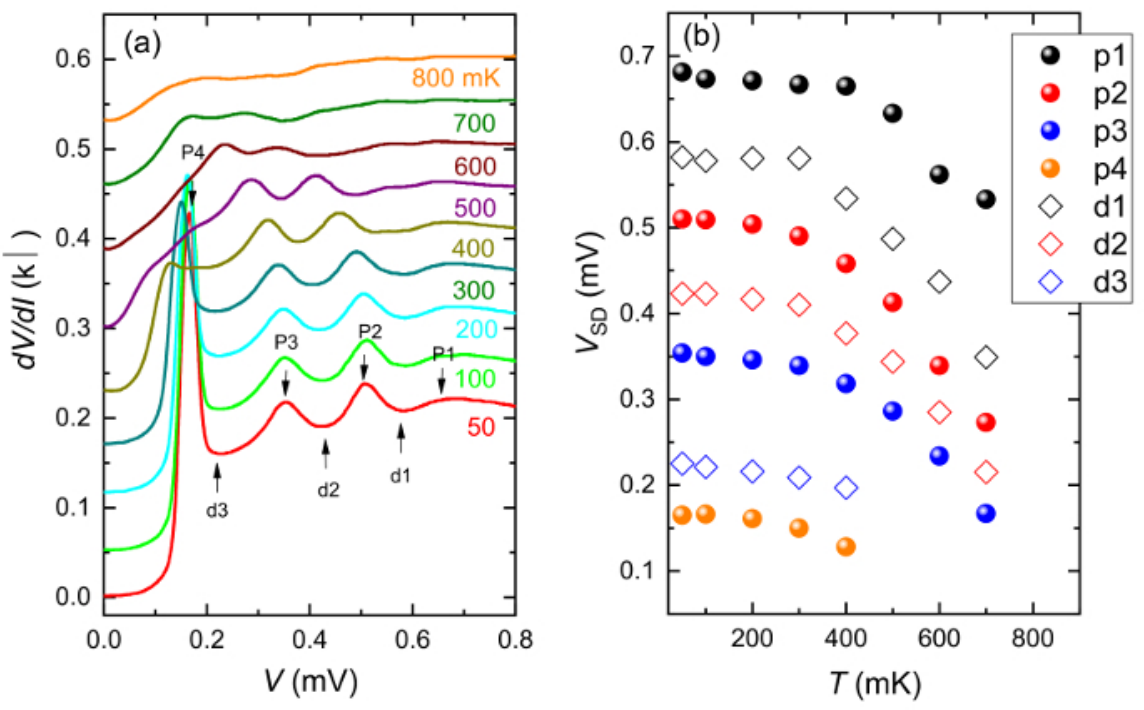

Figure 4. Induced superconductivity and $S G S$ in $\ln _{0.75} G a_{0.25} A s$ quantum wells in photolithographically fabricated junction. (a) Temperature dependence induced superconducting gap with pronounced SGS peaks due to multiple Andreev reflections. The SGS and the induced gap edge peaks, are marked by P1 to P4 while the SGS dips are marked by d1 to d3. (b) The SGS peaks and dips shown in (a) as a function of temperature. SGS are suppressed significantly at $T>400 \mathrm{mK}$ leading to a shift toward zero bias. Please click here to view a larger version of this figure. 

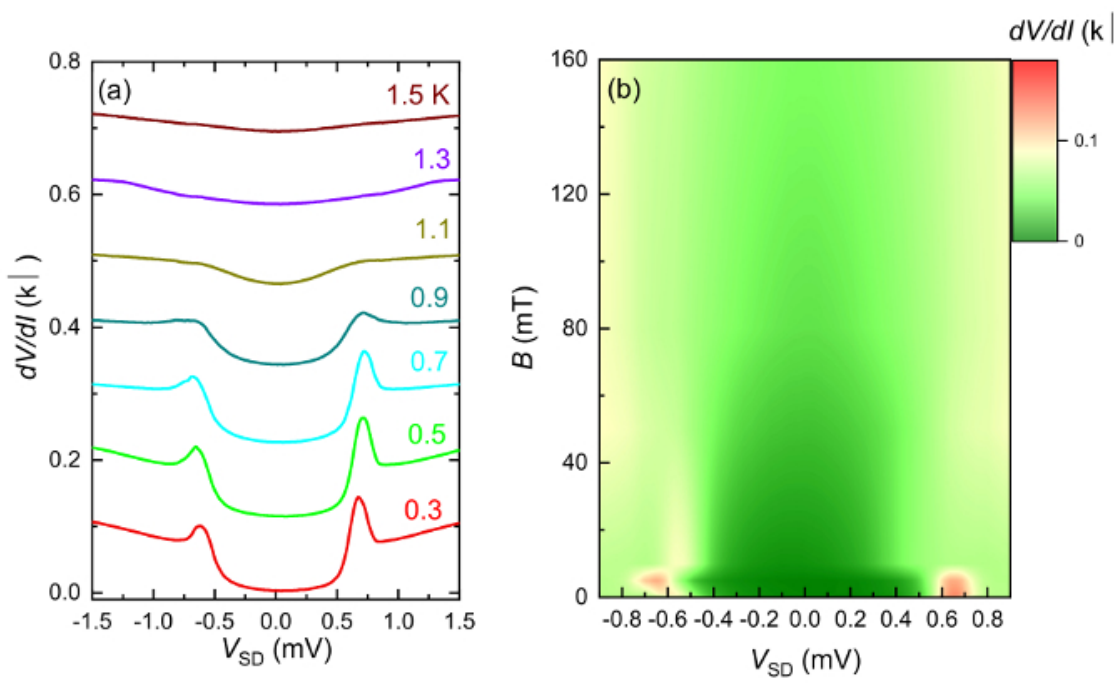

Figure 5. The temperature and magnetic field dependence of induced superconductivity in e-beam lithographically fabricated junctions. (a) Induced superconducting gap vs. applied source-drain voltage $V_{\mathrm{SD}}$ at temperatures between $300 \mathrm{mK}$ and $1.5 \mathrm{~K}$. The curves are vertically offset for clarity. (b) Color-coded differential resistance as a function of $V_{S D}$ and perpendicular magnetic field at $T=300 \mathrm{mK}$. Please click here to view a larger version of this figure.

\section{Discussion}

On-chip QICs comprising an array of JJs based on superconducting indium gallium arsenide $\left(\mathrm{In}_{0.75} \mathrm{Ga}_{0.25} \mathrm{As}\right)$ quantum wells were demonstrated. Two important challenges of hybrid S-Sm material systems such as the scalability and interface transparency were addressed. Two critical steps whining the protocol including the growth of high quality and high mobility $\ln _{0.75} \mathrm{Ga}_{0.25}$ As two-dimensional electron gas in semiconducting heterostructures and proximity induced superconductivity into $2 \mathrm{DEG}$ were discussed ${ }^{23,24,25}$.

Growth of $\operatorname{In}_{0.75} \mathrm{Ga}_{0.25}$ As with step-graded buffer layers in GaAs substrate and also the formation of homogeneous and barrier-free interfaces between the superconductor and semiconductor is a crucial step in such hybrid 2D quantum circuit development. It was demonstrated that with careful etching the sputtered superconducting film can make highly transparent contacts to $\ln _{0.75} \mathrm{Ga}_{0.25}$ As quantum wells resulting in detection of induced superconducting gap in semiconductors ${ }^{23,24,25}$.

The significance with respect to existing methods is that the presented technique for $2 \mathrm{D}$ hybrid JJs and circuit realization does not require the insitu deposition of superconductor on semiconductors in an MBE chamber after the semiconductor growth has been completed ${ }^{23,24,25}$. The other significance is that the heterostructure wafer can be grown as a desk of up to $10 \mathrm{~cm}$ diameter, allowing the fabrication of thousands of hybrid $2 \mathrm{D}$ junctions and circuits, so overcoming the scalability challenges of the hybrid S-Sm quantum circuits and devices ${ }^{22,23,24,25}$.

The induced superconductivity in quantum wells, SGS on differential conductance of the 2D junctions, and the phase coherent ballistic quantum transport measured in our junctions strongly suggest that hybrid $2 \mathrm{D}$ junctions and circuits based on superconducting $\mathrm{In}_{0.75} \mathrm{Ga}_{0.25} \mathrm{As} 2 \mathrm{DEG}$ afford promising material system for scalable quantum processing and computing technologies. Our approach may open a new road toward quantum technology and helps pave the way for the development of on-chip topological quantum circuits for realizing the next generation of quantum processors $^{23,24,25}$.

\section{Disclosures}

The authors have nothing to disclose.

\section{Acknowledgments}

The authors acknowledge financial support from EPSRC, grant MQIC.

\section{References}

1. Josephson, B. D., Possible new effects in superconductive tunneling, Physics Letters. 1, $251-253$ (1962).

2. Mukhanov, O. A. Energy-efficient single flux quantum technology, IEEE Transaction on Applied Superconductivity. 21, 760-9 (2011).

3. Tsujimoto, M., et al. Broadly Tunable Subterahertz Emission from Internal Branches of the Current-Voltage Characteristics of Superconducting $\mathrm{Bi}_{2} \mathrm{Sr}_{2} \mathrm{CaCu}_{2} \mathrm{O}_{8+\delta}$ Single Crystals, Physical Review Letters. 108 (10), 1-5 (2012).

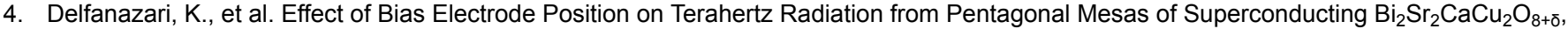
IEEE Transaction Terahertz Science and Technology. 5 (3), 505-511, (2015). 
5. Delfanazari, K., et al. Terahertz Oscillating Devices Based upon the Intrinsic Josephson Junctions in a High Temperature Superconductor. Journal of Infrared, Millimeter, Terahertz Waves. 35 (1), 131-146 (2014).

6. Delfanazari, K., et al. Tunable Terahertz Emission from the Intrinsic Josephson Junctions in $\mathrm{Acute}$ Isosceles $\mathrm{Triangular}^{\mathrm{Bi}} \mathrm{Si}_{2} \mathrm{CaCu}_{2} \mathrm{O}_{8+\delta}$ Mesas, Optics Express. 21 (2), 2171-2184 (2013).

7. Delfanazari, K., et al. Study of Coherent and Continuous Terahertz Wave Emission in Equilateral Triangular Mesas of Superconducting $\mathrm{Bi}_{2} \mathrm{Sr}_{2} \mathrm{CaCu}_{2} \mathrm{O}_{8+\delta}$ intrinsic Josephson Junctions. Physica C Superconductivity and its Application. 491, 16-19 (2013).

8. Kashiwagi, T., et al. High Temperature Superconductor Terahertz Emitters: Fundamental Physics and Its Applications, Japanese Journal of Applied Physics. 51 (1), 1- 14 (2012).

9. Klemm, R. A., et al. Modeling the Electromagnetic Cavity Mode Contributions to the $\mathrm{THz}$ Emission from $\mathrm{Triangular}^{\mathrm{Bi}} \mathrm{Sr}_{2} \mathrm{CaCu}_{2} \mathrm{O}_{8+\delta} \mathrm{mesas}_{\text {, }}$ Physica C Superconductivity and its Application. 491, 30-34 (2013).

10. Cerkoney, D. P., et al. Cavity Mode Enhancement of Terahertz Emission from Equilateral Triangular Microstrip Antennas of the HighTcsuperconductor $\mathrm{Bi}_{2} \mathrm{Sr}_{2} \mathrm{CaCu}_{2} \mathrm{O}_{8+\delta}$, Journal of Physics: Condensed Matter. 29 (1), 15601 (2017).

11. Sand-Jespersen, T., et al. Kondo-Enhanced Andreev Tunneling in InAs Nanowire Quantum Dots. Physical Review Letters. 99, 126603 (2007).

12. Herr, Q. P., et al. Reproducible operating margins on a 72800-device digital superconducting chip, Superconductor Science and Technology. 28, 124003 (2015).

13. Van Dam, J. A., Nazarov, Y. V., Bakkers, E. P. A. M., Franceschi, S. D., Kouwenhoven, L. P. Supercurrent reversal in quantum dots, Nature. 442, 667-670 (2006)

14. Giazotto, F., et al. A Josephson Quantum Electron Pump, Nature Physics. 7, 857-861 (2011).

15. Cybart, S.A., et al Large voltage modulation in magnetic field sensors from two dimensional arrays of YBaCuO nano Josephson junctions Applied Physics Letters. 104, 062601(2014).

16. Kalhor, S., Ghanaatshoar, M., Kashiwagi, T., Kadowaki, K., Kelly, M. J., Delfanazari, K. Thermal Tuning of High- Tc Superconducting $\mathrm{Bi}_{2} \mathrm{Sr}_{2} \mathrm{CaCu}_{2} \mathrm{O}_{8+\delta}$ Terahertz Metamaterial. IEEE Photonics Journal. 9 (5), 1-8 (2017).

17. Mourik, V., et al., Signatures of Majorana fermions in hybrid superconductor-semiconductor nanowire devices. Science. 336, 1003-1007 (2012).

18. Chang, W., et al. Hard gap in epitaxial semiconductor-superconductor nanowires, Nature Nanotechnology. 10, 1038 (2014).

19. Rokhinson, L. P., Liu, X., Furdyna, J. K., The fractional ac. Josephson effect in a semiconductor-superconductor nanowire as a signature of Majorana particles. Nature Physics. 8, 795-799 (2012).

20. Deng, M. T., et al., Majorana bound state in a coupled quantum-dot hybrid-nanowire system, Science. 354, 1557-1562 (2016).

21. Gül, Ö., et al. Hard Superconducting Gap in InSb Nanowires. Nano Letters. 17 (4), 2690-2696 (2017).

22. Nichele, F., et al. Scaling of Majorana Zero-Bias Conductance Peaks, Physical Review Letters. 119, 136803 (2017)

23. Delfanazari, K., et al. On Chip Andreev Devices: hard Gap and Quantum Transport in Ballistic Nb-In ${ }_{0.75} \mathrm{Ga}_{0.25} \mathrm{As}$ quantum well-Nb Josephson junctions, Advanced Materials. 29, 1701836 (2017).

24. Delfanazari, K., et al. Induced superconductivity in indium gallium arsenide quantum well, Journal of Magnetism and Magnetic Materials. 459, 282-284 (2018).

25. Delfanazari, K., et al. On-chip hybrid Superconducting-Semiconducting Quantum Circuit, IEEE Transactions on Applied Superconductivity. 28 4 (2018).

26. Chen, C., et al. Growth variations and scattering mechanisms in metamorphic $\ln _{0.75} \mathrm{Ga}_{0.25} A s / \mathrm{In}_{0.75} \mathrm{Al}_{0.25} \mathrm{As}$ quantum wells grown by molecular beam epitaxy, Journal of Crystal Growth. 425, 70-75 (2015).

27. Blonder, G. E., Tinkham, M., and Klapwijk, T. M., Transition from metallic to tunneling regimes in superconducting micro-constrictions: Excess current, charge imbalance, and supercurrent conversion, Physical Review B. 25, 4515 (1982).

28. Beenakker, C. W. J., Random-matrix theory of quantum transport, Review Modern Physics. 69, 731 (1997). 